\title{
Application of Digitization in Offshore Oilfield
}

\author{
Xiaojiao Tong \\ CNOOC Ltd.-Tianjin, Tianjin, China \\ Email: tongxj@cnooc.com.cn
}

How to cite this paper: Tong, X.J. (2021) Application of Digitization in Offshore Oilfield. Open Journal of Applied Sciences, 11, 1009-1015. https://doi.org/10.4236/ojapps.2021.119073

Received: August 24, 2021

Accepted: September 13, 2021

Published: September 16, 2021

Copyright $\odot 2021$ by author(s) and Scientific Research Publishing Inc. This work is licensed under the Creative Commons Attribution International License (CC BY 4.0).

http://creativecommons.org/licenses/by/4.0/

\section{(c) (i) Open Access}

\begin{abstract}
With the rapid development of information technology, the systematization and informatization of equipment management has become the goal of world advanced enterprises. With the rapid development of offshore oil fields, equipment maintenance management and safety control have been promoted to a new level. With the rapid growth of the company's business and asset scale, the system users have increased year by year, the functions have been deepened and applied, and the investment in asset management informatization has also increased year by year. By analyzing the actual situation of personnel shift management on offshore platform, combined with the advantages of equipment personnel management in the system framework, this paper makes a digital exploration. Take informatization as the support, innovate working methods, improve management efficiency, and create intelligent shift handover, so as to achieve the purpose of improving quality and efficiency. Improve system application efficiency, business tightness and management efficiency.
\end{abstract}

\section{Keywords}

Maximo, Shift Management, Public Account Optimization, Equipment Management System, Single Sign-On

\section{Introduction}

With the rapid development of information technology, the systematization and informatization of equipment management has become the goal of world advanced enterprises. Maximo system is an asset management software developed by IBM of the United States. It is an enterprise information system application platform with equipment maintenance as the core. Maximo is the most widely used enterprise asset management system in the market. It is a world-class asset management system. It has scientific maintenance management of asset intensive enterprises and standard processes and experience of surrounding business- 
es. Maximo can achieve the purpose of improving the safety and efficiency of workers and making full use of human and other resources in Enterprise management [1] [2] [3] [4] [5].

With the rapid development of offshore oil fields, equipment maintenance management and safety control have been promoted to a new level. With the rapid growth of the company's business and asset scale, the system users have increased year by year, the functions have been deepened and applied, and the investment in asset management informatization has also increased year by year. How to further improve the system application efficiency, business tightness and management efficiency has become the main problem faced by the management of enterprise information department. Based on this, we take informatization as the support, innovate working methods, improve management efficiency, and create intelligent shift handover, so as to achieve the purpose of improving quality and efficiency [6] [7] [8] [9].

\section{Management Method Practice Based on Maximo}

The offshore oil field platform has been in-depth information construction. Based on the mature conditions of network environment, safety control and unified planning, a number of information operation systems have been built and invested according to the tightness of production work, so as to ensure the steady development of the overall level of enterprise information construction year by year, including equipment management in production and operation links. The unified Maximo system within the group is adopted. At the same time, it optimizes the management of Maximo users and saves resources. Through the use of the system for the scientific maintenance management of asset intensive enterprises and the standard process and experience of surrounding businesses, combined with the customized development of the self operated oilfield of Tianjin Branch for more than ten years, each business module has formed an equipment management application and process with CNOOC characteristics.

\subsection{Establishment of Open Web Architecture}

Maximo is a real Web architecture product. It adopts n-tier, Java based component architecture. On the server side, database layer, application layer and presentation layer, each layer can be distributed on multiple physical servers. With the improvement of server performance requirements, it can be expanded horizontally and vertically. In addition to supporting integration at the database level, Maximo can integrate other systems in a simpler, cheaper and more seamless way, that is, through commercial components and Maximo (Figure 1).

\subsection{Implementation of Maximo User Single Sign-On}

The mapping relationship between the domain account and the Maximo user is established in the Maximo system. The Maximo server realizes the function of 


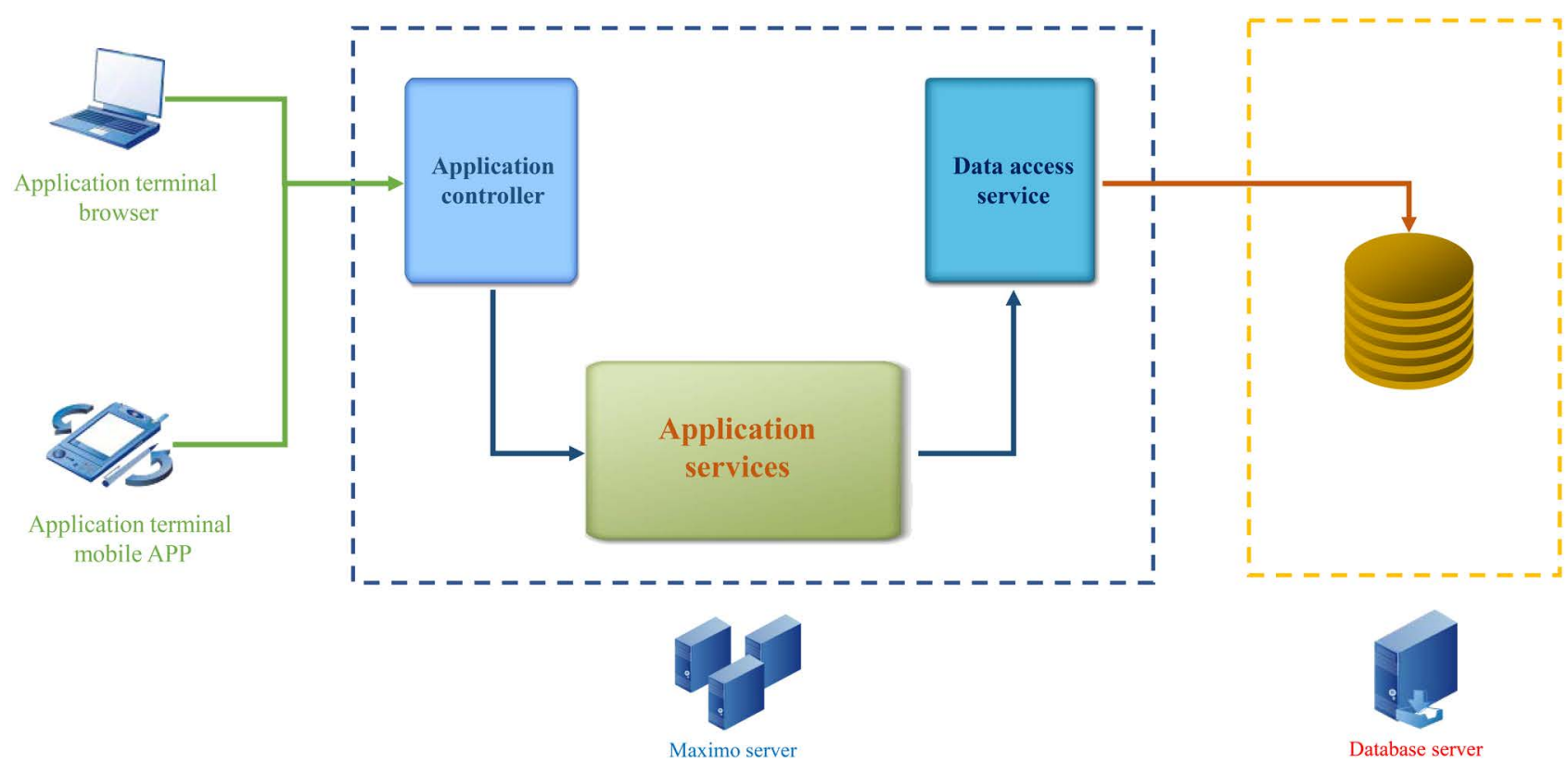

Figure 1. Web architecture integration module.

verifying the client domain account information through customized development. After confirming the feedback domain account information, access the Maximo database to retrieve whether it matches the Maximo user information. Through the logical processing of domain account mapping Maximo users, the function of directly logging in to Maximo system is realized.

\subsection{Business Process Optimization Based on Maximo}

Develop "shift management" application function in Maximo system resource module. In order to facilitate offshore employees to fill in relevant shift information and authorize Maximo users to establish an application of "shift management" for other employees in the resource module. The application contains relevant information such as users and personnel. Users can associate with user names by selecting personnel and saving them. The business operation of re logging into the system with the Maximo user is to select the employee system and record the operation history.

By creating and managing user records. User records contain user names, passwords, and security profiles that determine which applications, options, and data users can access. When creating user records, you must also create personnel records. A user record can only be associated with one person record. A person record can only be associated with one user record. You can associate a manual record and a user record with the same person record. By default, this directory manages user creation when using the application server for authentication. You can set properties to perform user creation directly in the system. By setting these properties, you can enable or disable some features in the system. The relevant data sheets are updated as follows:

Maxuser: update with new user data. 
Person: update with new person data (if you need to create a person record for a new user).

Phone: store all user phone number information, including home phone number, work phone number, mobile phone number, pager number, etc. indicates the primary phone number.

Email: stores all user e-mail addresses, including home e-mail address, work e-mail address and alternate e-mail address. Indicates the primary email address

Groupuser: updates the association between security groups and users.

Userpurgl: stores the default purchase general ledger account for new users.

Grpreassignauth: stores the user name of the person who is authorized to add users to the security group.

Maxuserstatus: displays the status of the user, for example, active or blocked; stores a history of user status changes.

Password Directory: stores the user's current password and the history of password changes. If password duration is enabled, the data in the password column is encrypted.

Local Maximo validation (Figure 2):

1) On the Maximo web client login screen, the user enters the login ID (in the user name field) and password.

2) The Maximo security service verifies the user's credentials against the Maximo database. This process checks the user in the Maximo user mode/database

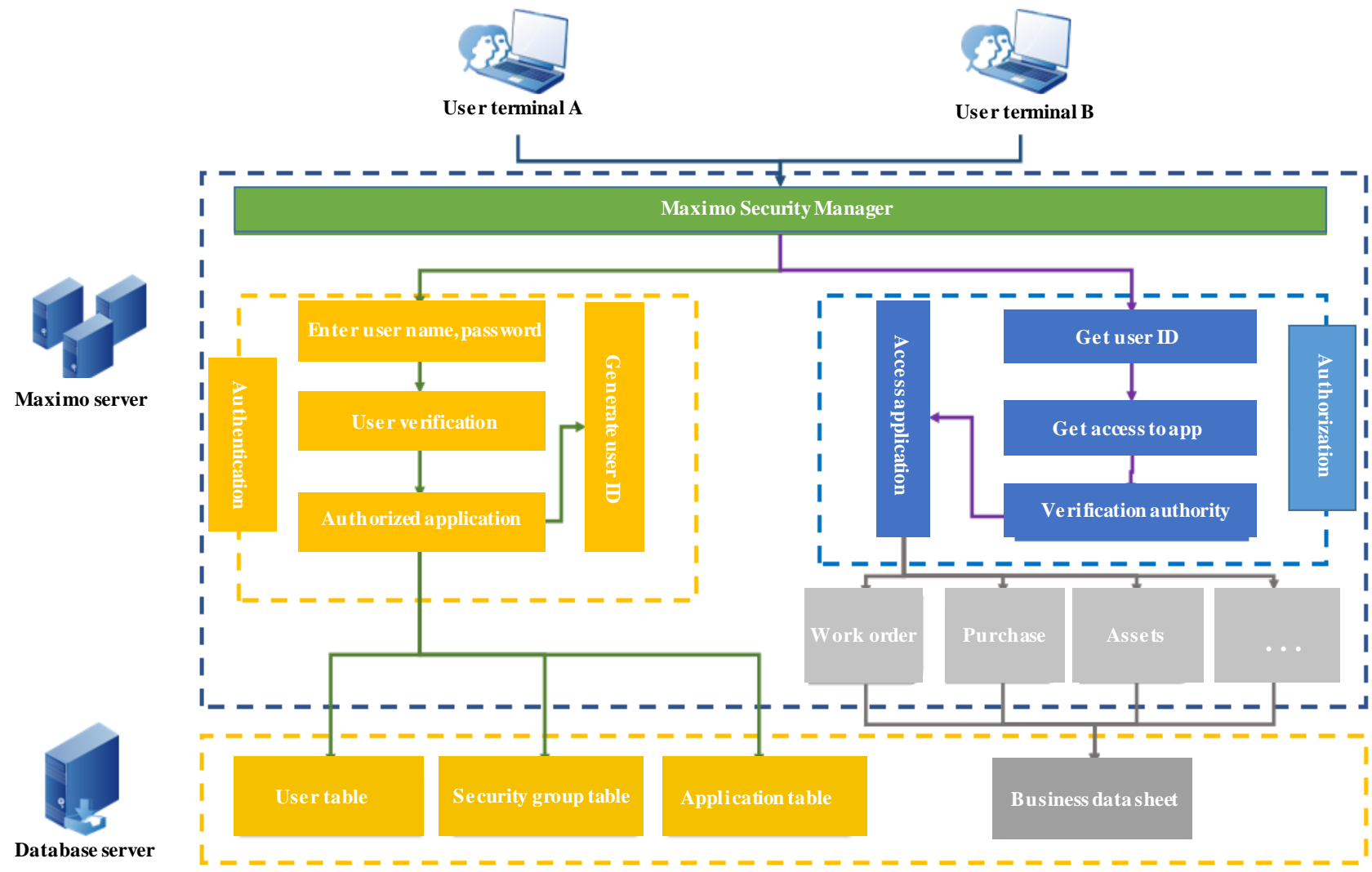

Figure 2. Validation authorization logic diagram after optimization. 
using encryption method.

3) The system checks the user's permission profile. According to the authorization contained in the configuration file, the system grants the user access to the Maximo application.

\subsection{Personnel Handover Application Scenario}

The standard function of Maximo system is to use the "security group" application to set and manage users' security privileges. Users can be assigned to groups through the security groups application and the users application.

In the security groups application, people can assign users to groups. In the users application, shift personnel can assign groups to users. Shift personnel can use the security profile tab in the user application to view the user's security privileges.

By creating and managing user records. User records contain user names, passwords, and security profiles that determine which applications, options, and data users can access. When creating user records, you must also create personnel records. A user record can only be associated with one person record. A person record can only be associated with one user record. You can associate a manual record and a user record with the same person record. By default, this directory manages user creation when using the application server for authentication. You can set properties to perform user creation directly in the system. By setting these properties, you can enable or disable some features in the system.

\section{Advantages and Effects of Digital Management}

\subsection{Accurate Information Management}

Before implementation, shift personnel need to hand over their work offline. After implementation, standardize the handover template, record the progress of matters to be supervised through the system according to the unified process, and connect the electronic accessories of important information in the work. The handover process and interface are clear and accurate. The intellectualization of the handover process has greatly improved the standardization of management. Through the data operation and recording function of Maximo system, the system data operator can accurately record the system data. With the continuous accumulation of information and data assets, it provides more potential to help the development of smart oilfield.

\subsection{Efficient Data Exchange}

When offshore personnel hand over work, log in to the application and select the personnel to be handed over. After saving, when logging in the system with the same user information, all business operations are recorded by the selected transferred personnel. You can also judge whether the association between users and employees is correct by the name in the upper left corner of the page. If it is inconsistent with the current employee, you can select the correct shift change 
employee through this application. After logging in the system again, confirm that the employee name displayed on the page is consistent with the current employee, that is, the shift handover is successful.

Add shift management procedures in the resource management module, apply logic independently, avoid the business coupling between the organizational structure and the assignment of personnel and shift employees, and enhance the expansion ability of shift related functions in the future. Change the function of filtering users (records and filtering) in the program to filter employees for the enterprise site: each application module in the system involves the filtering of foreground users, which has been enhanced to update the filtering users of the enterprise site to filter employees. Adjust the employee information recorded in the process of each module (work order, purchase, outsourcing, security, etc.).

\subsection{Fine Management Effect}

After the implementation of digital shift handover, it innovatively changed the previous extensive shift handover management mode, stepped into the modeling of fine management, and realized the informatization, standardization, process and refinement of the handover process. The system makes permission control and data operation records according to employee information, finely records on-the-job rights and responsibilities, and the historical records can be traced. At the same time, the land management personnel can also query the names of the personnel on duty through the system at any time. The management is refined and the resource allocation is standardized. This is expected to reduce the idle rate of users by more than $50 \%$ in the long term. When the company's platform goes online and the investment volume of production and operation personnel increases in the future, especially with the expansion of the scale of the oil field, the efficiency will become more and more obvious.

\section{Conclusions}

Through the practice of digital management of personnel shift handover by using Maximo system, the actual effect is good. While completing the application of the system, reducing cost and increasing efficiency, it standardizes relevant management systems and improves management ability:

1) Establish the document and data management mechanism of shift handover, so that the shift handover documents at sea have been scientifically and effectively managed.

2) Bring the offshore shift management into the unified management of the system. The tracking and supervision mechanism of offshore shift handover information is established to make the task allocation and assignment clearer;

3) Realize the single point landing of offshore Maximo system landing, and improve the work efficiency of users on the basis of providing users with better experience.

4) It reduces the number of Maximo users for the company and directly saves 
costs for the enterprise.

\section{Conflicts of Interest}

The author declares no conflicts of interest regarding the publication of this paper.

\section{References}

[1] Li, Y. (2010) Design of Performance Management System Based on Maximo Platform. Inner Mongolia Power Technology, No. 5, 37-39.

[2] Mu, L. (2017) Research on Extended Application of Maximo System in PY Oilfield Oil Platform. Tianjin University, Tianjin.

[3] Chen, J., He, G. and Wen, H. (2008) Construction of Material Management System for Electric Power Enterprises Based on Maximo. Electric Power Informatization, No. 3, 80-83.

[4] Zhang, P. (2019) Exploration on the Extension of EAM System to Industrial Internet of Things. Resource Conservation and Environmental Protection, No. 5, 121-123.

[5] El Khatib, M. and Ahmed, G. (2018) Improving Efficiency in IBM Asset Management Software System "Maximo": A Case Study of Dubai Airports and Abu Dhabi National Energy Company. Theoretical Economics Letters, 8, 1816-1829. https://doi.org/10.4236/tel.2018.810119

[6] Chen, R. (2019) Design and Implementation of Material Management System Based on Maximo Platform. University of Electronic Science and Technology of China, Chengdu.

[7] Ma, W. and Wang, X. (2021) How to Use Shift Handover and Yard Day to Improve "Five Knowledge and One Ability" of Commanders and Fighters in Grass-Roots Team Stations. Fire Industry (Electronic Version), No. 11, 42+44.

[8] Wang, L. (2001) Introduction to Maximo Enterprise Asset Management Software. Equipment Management and Maintenance, No. 6, 6-8.

[9] Yuan, C., Meng, X. and Li, H. (2006) Analysis on the Management Idea of Maximo System Architecture. Journal of Shandong Electric Power College, No. 2, 103-104. 